\title{
Genetic diversity of sea-island cotton (Gossypium barbadense) revealed by mapped SSRs
}

\author{
X.Q. Wang', C.H. Feng ${ }^{1,2}$, Z.X. Lin ${ }^{1}$ and X.L. Zhang ${ }^{1}$ \\ ${ }^{1}$ National Key Laboratory of Crop Genetic Improvement, \\ National Centre of Plant Gene Research, Huazhong Agricultural University, \\ Wuhan, Hubei, China \\ ${ }^{2}$ Institute of Industrial Crops, Hubei Academy of Agricultural Sciences, \\ Wuhan, Hubei, China \\ Corresponding author: Z.X. Lin \\ E-mail: linzhongxu@mail.hzau.edu.cn
}

Genet. Mol. Res. 10 (4): 3620-3631 (2011)

Received April 27, 2011

Accepted October 26, 2011

Published December 8, 2011

DOI http://dx.doi.org/10.4238/2011.December.8.5

\begin{abstract}
In order to evaluate the genetic diversity of seaisland cotton (Gossypium barbadense), 237 commonly mapped SSR markers covering the cotton genome were used to genotype 56 seaisland cotton accessions. A total of 218 polymorphic primer pairs $(91.98 \%)$ amplified 361 loci, with a mean of 1.66 loci. Polymorphism information content values of the SSR primers ranged from 0.035 to 0.862 , with a mean of 0.320 . The highest mean polymorphism information content value for the SSR motifs was from a compound motif (0.402), and for the chromosomes it was Chr10 (0.589); the highest ratio of polymorphic primers in Xinjiang accessions was from Chr21 (83.33\%). Genetic diversity was high in Xinjiang accessions. AMOVA showed that variation was 8 and 92\% among populations and within populations, respectively. The 56 sea-island accessions were divided into three groups in the UPGMA dendrogram: Xinhai5 was in the first group; accessions from Xinjiang, except the five main ones, were in the second group, and the other 34 accessions
\end{abstract}


were in the third group. Accessions from the former Soviet Union and Xinjiang main accessions were closely related. Both PCA and UPGMA confirmed that Xinhai5 was distinct from the other accessions, and accessions from Xinjiang were in an independent group. Given the differences between principal components analysis and UPGMA results, it is necessary to combine molecular markers and pedigree information so that genetic diversity can be objectively analyzed.

Key words: Sea-island cotton; Genetic diversity; Mapped SSR

\section{INTRODUCTION}

Cotton (Gossypium spp), a unique cash crop that produces fiber from seed, provides the world's largest number of natural textile fibers. The cotton genus Gossypium L. consists of about 45 diploid and five tetraploid species (Fryxell, 1992). Among the five tetraploid species, two species have been domesticated. They are G. hirsutum L. (predominantly distributed in Meso-America and the Caribbean) and G. barbadense L. (mainly distributed in South America and the Caribbean) (Westengen et al., 2005). Upland cotton (G. hirsutum) and sea-island cotton (G. barbadense) contribute to 90 and $8 \%$ of the world's cotton production, respectively (Song et al., 2004).

Sea-island cotton is tall and robust, with large leaves and a long life period. Its prominent feature is its perfect fiber quality. Besides, this species has many other important traits, such as cold tolerance, drought tolerance, disease resistance, and insect resistance (Mehetre et al., 2003), which are absent in the high-yield upland cotton. Sea-island cotton includes annual and perennial types, and the annual ones are mainly cultivated. Cultivated annual sea-island cotton is classified into three ecotypes: Egyptian type, American type and Middle-Asia type (Mei et al., 2001). Annual sea-island cotton was introduced into China in 1954, and it has been experimentally planted in Yunnan, Guangxi, Guangdong, Fujian, Shanghai, Henan, Jiangsu, Xinjiang, etc. (Kong, 2002). Now, Xinjiang is the only growing region where sea-island cotton is planted. Besides the introduced cultivars, cotton breeders in China have developed several new sea-island cotton cultivars with high-yield production and good fiber quality, for example, Junhai 1 and Xinhai series cultivars.

Several molecular markers such as AFLP (Rana et al., 2005; Westengen et al., 2005), RAPD (Vafai-Tabar et al., 2004), SSR (Lacape et al., 2007; Kantartzi et al., 2009; Luo et al., 2010) and ISSR (Dongre et al., 2004) have been used in upland cotton germplasm analysis, which is helpful to reveal the genetic diversity of these varieties in future breeding. Molecular markers such as SSR (Diao et al., 2002; Chen et al., 2005; Li et al., 2009; Wu et al., 2010), ISSR (Jiang et al., 2008) and SRAP (Li et al., 2008) have also been applied in determining the genetic diversity of sea-island cotton.

In our previous study, Li et al. (2008) used SRAP markers to analyze the genetic diversity of sea-island cotton accessions from China and other countries. Because the chromosome assignment of SRAP remains unknown, it is hard to understand the genomic diversity of these accessions. In recent years, more than 10,000 SSR markers have been released (CMD website, http://www.cottonmarker.org) and mapped in cotton (Nguyen et al., 
2004; Rong et al., 2004; Frelichowski Jr. et al., 2006; Guo et al., 2007; He et al., 2007; Yu et al., 2007; Zhang et al., 2008). In this study, to better understand the population structure of sea-island cotton in China, we used commonly mapped SSR markers to study the genetic diversity of sea-island cotton.

\section{MATERIAL AND METHODS}

\section{Plant material}

The 56 sea-island cotton accessions used in this study were those previously studied by Li et al. (2008), including the cultivars cultivated in different regions in China and some accessions introduced from foreign countries. DNAs used in this study were from Li et al. (2008).

\section{SSR analysis}

SSR markers were selected from the seven most informative and high-density interspecific linkage maps of cotton (Rong et al., 2004; Nguyen et al., 2004; Frelichowski Jr. et al., 2006; He et al., 2007; Guo et al., 2007; Yu et al., 2007; Zhang et al., 2008). The selection criteria were that 1) SSR markers were mapped in at least three maps and 2) a single locus was detected by each SSR. A total of 237 pairs of SSRs were selected from 25 chromosomes with 4 to 17 markers from each chromosome (Supplementary Table 1). No markers were selected from $\mathrm{Chr} 22$ because they amplified multiple loci on other chromosomes.

PCR mixtures $(10 \mu \mathrm{L})$ consisted of $1 \mathrm{X}$ reaction buffer, $1.5 \mathrm{mM} \mathrm{MgCl}_{2}, 0.2 \mathrm{mM}$ dNTP, $0.3 \mu \mathrm{M}$ primers, $25 \mathrm{ng}$ template DNA, and $0.5 \mathrm{U}$ Taq DNA polymerase (MBI). Amplification was carried out in a BIO-RAD thermal cycler, with an initial $5 \mathrm{~min}$ at $94^{\circ} \mathrm{C}$, which was followed by 34 cycles of $1 \mathrm{~min}$ at $94^{\circ} \mathrm{C}, 1 \mathrm{~min}$ at $\mathrm{X}^{\circ} \mathrm{C}$, and $1 \mathrm{~min}$ at $72^{\circ} \mathrm{C}$, plus a 5-min final extension at $72^{\circ} \mathrm{C} . \mathrm{X}^{\circ} \mathrm{C}$ refers to the annealing temperature specified for each primer. The amplified products were separated on $6 \%(\mathrm{w} / \mathrm{v})$ denaturing polyacrylamide $(1: 19$ bis:acrylamide) gels and visualized with silver staining.

\section{Data analysis}

SSR fragment was coded as "1" for presence, "0" for absence and "9" for missing data. An original data matrix was then generated for further study. PIC (polymorphism information content) was calculated according to Zhang et al. (2007).

The genetic diversity statistics of sea-island cotton was analyzed using the frequency procedure of the GenAlEx6.2 software (Cruz et al., 2007), including: sample size, number of different alleles, number of effective alleles, Shannon's information index, and expected heterozygosity. Analysis of molecular variance (AMOVA) was used to estimate molecular diversity at the hierarchical level between and within populations, and principal coordinates analysis (PCA) was based on Nei's genetic distance.

Genetic similarity coefficient matrices were obtained by SIMQUAL of the NTSYS-pc 2.1 statistics package (Rohlf, 2000) based on Jaccard's algorithm. The similarity coefficient was used to construct a genetic distance matrix using the SAHN procedure based on unweighted pairgroup method analysis (UPGMA). 


\section{RESULTS}

\section{Amplification efficiency of SSRs}

The 237 SSR primer pairs yielded 381 loci; among them, 218 primer pairs (91.98 \%) generated 361 polymorphic loci with an average of 1.66 loci per primer pair (Supplementary Table 2).

PIC values of primers ranged from 0.035 to 0.862 with a mean of 0.320 . The higher average PIC values of SSR repeat motifs were: compound ( 0.402$)$, interrupted $(0.373)$ and trinucleotide $(0.369)$ (Table 1). When taking chromosomes into account, the higher average PIC values of the chromosomes were for Chr10 (0.589), followed by Chr21 (0.465) and Chr04 (0.438) (Supplementary Table 2).

Table 1. Amplification of different SSR repeat motifs.
\begin{tabular}{lccccc}
\hline SSR repeat motif & Markers & Polymorphic rate (\%) & Average amplified loci & Range of PIC & Mean PIC \\
\hline Dinucleotide & 179 & 92.18 & 1.66 & $0.035-0.855$ & 0.342 \\
Trinucleotide & 9 & 77.78 & 1.71 & $0.035-0.762$ & 0.369 \\
Pentanucleotide & 1 & 100.00 & 1.00 & $0.035-0.035$ & 0.035 \\
Hexanucleotide & 1 & 100.00 & 1.00 & $0.035-0.777$ & 0.035 \\
Compound & 10 & 90.00 & 1.67 & $0.035-0.862$ & 0.373 \\
Interrupted & 37 & 94.59 & 1.63 & & \\
\hline
\end{tabular}

\section{Assessment of genetic diversity statistics}

In order to easily analyze the genetic diversity of 56 sea-island cotton accessions, we classified them based on their origins. Numbers 1-14 and 24-29 are foreign accessions, as Pop1; numbers 15-23, as Pop2, are domestic cultivars, which are mainly from the Yangtzi River region, Yellow River region and Southern cotton region, and numbers 30-56 are the cultivars developed in Xinjiang, as Pop3.

Genetic diversity statistics showed that the order of all statistic values was Pop3 > Pop1 > Pop2; moreover, Pop1 and Pop2 had similar statistic values (Table 2).

Table 2. Comparison of genetic diversity statistics of three pops.
\begin{tabular}{lccccc}
\hline Pop type & Polymorphic (\%) & $N_{\mathrm{A}}$ & $N_{\mathrm{E}}$ & $\mathrm{I}$ & $H_{\mathrm{E}}$ \\
\hline Pop1 & 42.78 & 0.86 & 1.16 & 0.16 & 0.10 \\
Pop2 & 37.27 & 0.76 & 1.15 & 0.15 & 0.09 \\
Pop3 & 86.61 & 1.73 & 1.71 & 0.21 & 0.12 \\
\hline
\end{tabular}

$N_{\mathrm{A}}=$ number of different alleles; $N_{\mathrm{E}}=$ number of effective alleles; I = Shannon's information index; $H_{\mathrm{E}}=$ expected heterozygosity.

\section{AMOVA and PCA results}

AMOVA showed that $8 \%$ of the total molecular variance contributed to population divergence and $92 \%$ to individual differences. In the PCA graph (Figure 1), principal coordinates 1,2 and 3 explained 26.72, 22.59 and $16.01 \%$ of variation, respectively. The most direct view of Figure 1 is that 37 (Xinhai5), which belonged to Pop3, was separated from Pop3, and far away from other accessions. Moreover, genetic distance of Pop3 was much larger than that of Pop1 and Pop2. To some extent, Pop3 was an independent group; however, gene flow phenomenon was evident between Pop1 and Pop2. 


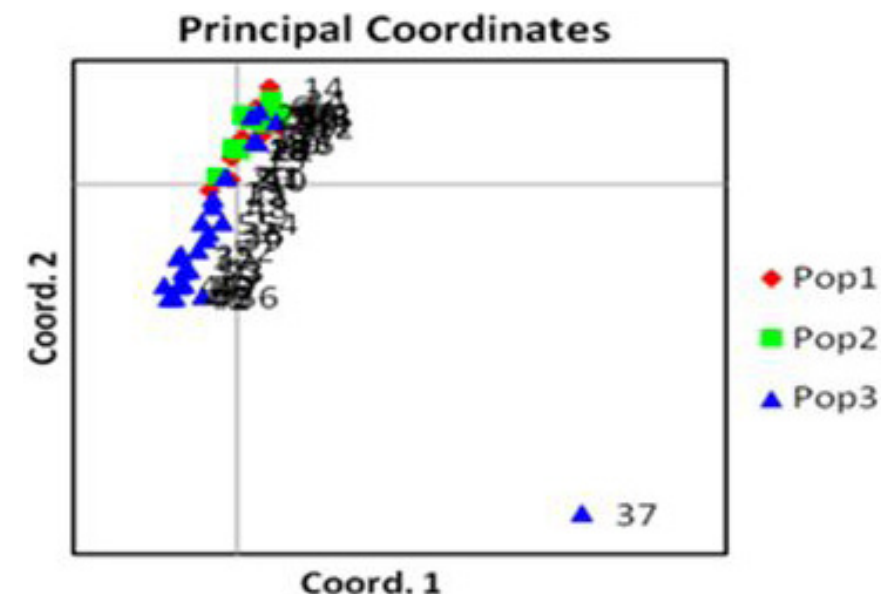

Figure 1. PCA graph of 56 sea-island cotton accessions based on SSRs. Red diamonds represented Pop1, green squares Pop2, and blue triangles Pop3.

\section{UPGMA analysis by SSRs}

Taking 0.22 as a threshold value, the 56 sea-island cotton accessions were divided into 3 groups (Figure 2). The first group was located at the bottom of the figure, only including 37 (Xinhai5). The second group was in the middle oval box including most of the latest cultivars from Xinjiang. The third group was in the upper oval box; this group included foreign accessions and other domestic cultivars except those followed by a dot, namely 30 (Shengli1), 31 (Tuhai2), 33 (Xinhaimian), 34 (Xinhai2), and 36 (Xinhai4), which are the basic cultivars in Xinjiang.

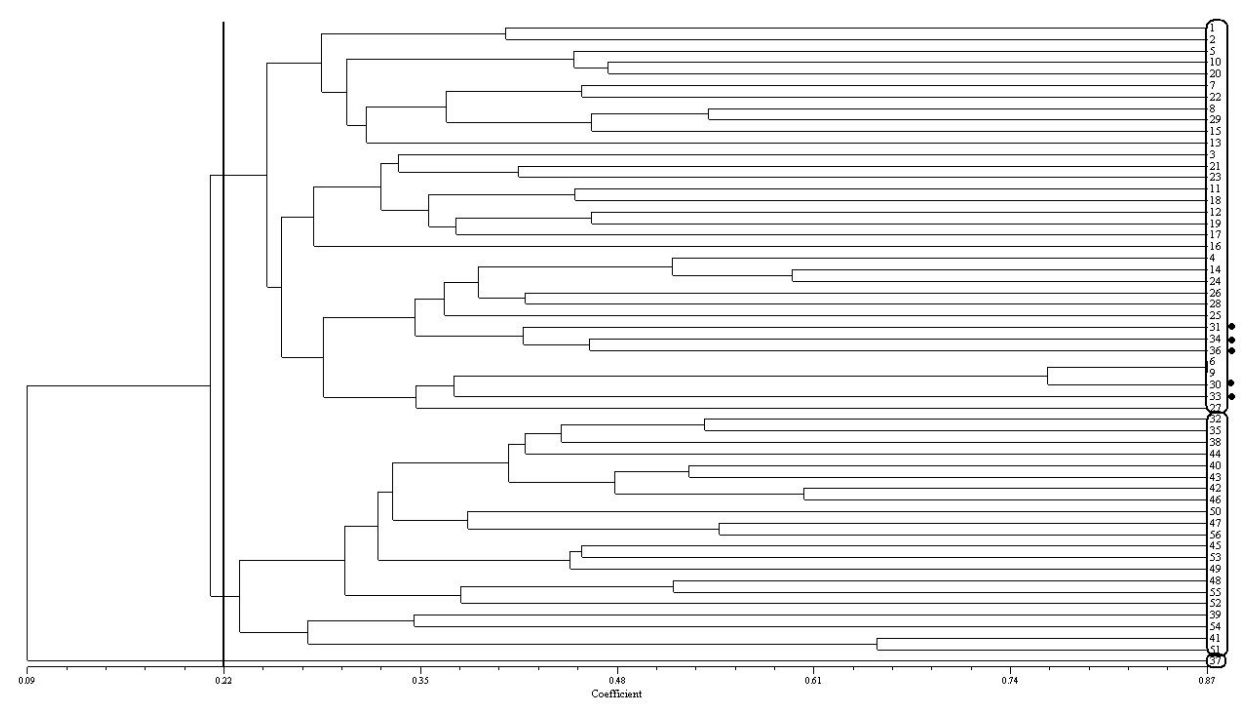

Figure 2. UPGMA dendrogram based on SSRs. Bold vertical line in the figure represents threshold lines. Oval boxes on the right represent different groups. The accessions are followed by a dot representing special individuals. 
In order to characterize the diversity of cultivars from Xinjiang (excluding 37 and 56), the polymorphic primers among accessions from Xinjiang were picked out to calculate the polymorphic ratio of the 25 chromosomes (Figure 3). The variations of accessions from Xinjiang were more on $\mathrm{Chr} 06, \mathrm{Chr} 08, \mathrm{Chr} 10$, and $\mathrm{Chr} 21$ with up to $70 \%$ of polymorphism on these chromosomes, and less than $30 \%$ on Chr07, Chr09, Chr11, and Chr17.

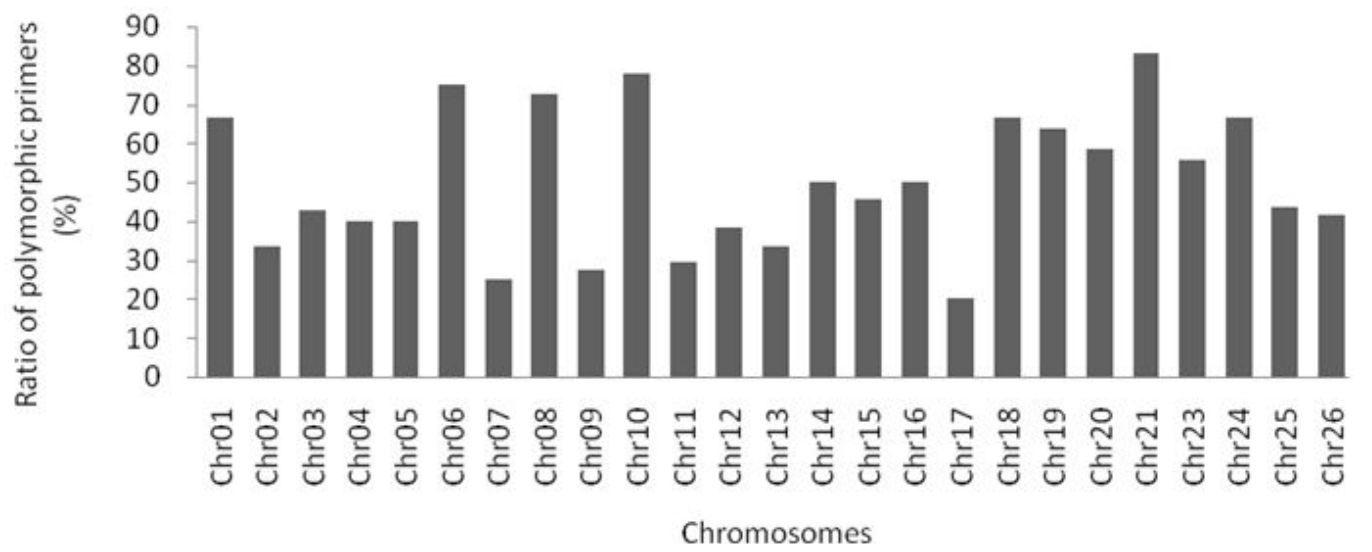

Figure 3. Ratio of polymorphic primers in Xinjiang cultivars except Xinhai5 and Xinhai25 on 25 chromosomes.

Comparing UPGMA trees and PCA results, we found some consistencies and differences. The consistencies included: 1) the latest cultivars from Xinjiang were clustered together, which was the same with the Pop3 results of PCA. 2) There were little differences between foreign and other domestic accessions, i.e., gene flow should exist, which was mainly induced by the introduction of foreign accessions. Differences included: 1) 37 (Xinhai5), which belonged to Pop3, was separated from the other 55 accessions in UPGMA results. 2) Basic cultivars, which belonged to Pop3, are clustered with the foreign ones in UPGMA results.

\section{DISCUSSION}

All 237 mapped SSRs were selected based on their distribution among 25 chromosomes. From Supplementary Table 2, we found that the higher average PIC values of the chromosomes were for Chr10 (0.589), followed by Chr21 (0.465) and Chr04 (0.438). However, there was no obvious relationship between the amplification efficiency of markers and chromosomes. Figure 3 showed that the ratios of polymorphic primers of Xinjiang accessions on Chr21 and Chr10 were larger than those on other chromosomes. From the above results, it can be deduced that the polymorphic information is rich on Chr21 and Chr10. This can be of great help in discovering genes related to the traits of sea-island cotton.

To some extent, PIC is a measure to detect the polymorphism of primers, which can be used to estimate the value of allele variation of each primer pair. PIC value of primers shows a positive relationship with the ability to reveal allelic variation (Rohlf, 2000). As shown in Table 1, higher average PIC values of SSR repeat motifs were: compound, interrupted, trinucleotide, and dinucleotide. Qureshi et al. (2004) reported a positive correlation between polymorphism and repeat number. Guo et al. (2007) found that the level of polymorphism appeared to be related to re- 
peat type. Zhang et al. (2008) found that the polymorphic rate of tetranucleotide repeats was higher than dinucleotide, trinucleotide, hexanucleotide, and pentanucleotide repeats. However, due to the limitation of different repeat motifs, except dinucleotide, the other five types of repeat motifs were fewer. Moreover, this study did not pick out SSRs of tetranucleotide repeat motifs. Therefore, the results reflected by this article had some limitations.

In this study, PIC analysis by SSR was carried out on 56 sea-island cotton accessions. The PIC values of SSR primers ranged from $0.035-0.862$, and the average was 0.320 . For SRAP markers, PIC values ranged from 0.035-0.896, with an average of 0.52 (Li et al., 2008). Obviously, the PIC value of SRAP was much higher than that of SSR. Moreover, the average polymorphic loci of SRAP was more than that of SSR (3.17vs 1.66), indicating that SRAP was superior to SSR in the ability of detecting polymorphism, which was also confirmed by other researchers (Zhang et al., 2007; Tan et al., 2009).

The study of genetic diversity can help us understand the genetic structure and polymorphism of sea-island cotton, and provide a theoretical basis for studying the origin and classification of these accessions, as well as parent selection and variety protection. Li et al. (2008) and Chen et al. (2005) showed that the cultivars from Xinjiang were more similar to foreign accessions. In this study, the genetic relationship between cultivars from Xinjiang was much more complex: the basic cultivars were closer with the ones from the former Soviet Union, but the latest cultivars were stable. One possible reason may be that although the basic cultivars were developed from some foreign accessions, in the later breeding stage, Xinjiang cultivars mainly came from several basic germplasms (such as Junhai1, Xinhaimian and Tuhai2), and in the cultivation process, these cultivars produced other variations which gradually became a special genetic structure due to the unique climate condition of Xinjiang region (Wang and Diao, 2002).

The high similarity coefficient between cultivars showed that genetic diversity of these sea-island cotton accessions from Xinjiang was narrow, which was consistent with the results of He et al. (2002) and Li et al. (2009). That is to say, the breeding of Xinhai series is likely to face a bottleneck effect just as upland cotton does (Iqbal et al., 2001). Therefore, new breeding methods and the introduction of new germplasm are necessary. It is worth noting that the genetic similarities between Xinhai5 and Xinhai25 were far from Xinjiang varieties as well as foreign and other domestic sea-island cotton cultivars. According to the pedigree, Xinhai5 includes genes of MiddleAsia type, Egyptian type sea-island cotton and mallow, and Xinhai25 was bred from the hybrid of many high-generation varieties ( $\mathrm{Li}$ et al., 2008). Another reason may be that genes were influenced by cultivation progress and environmental factors. Besides, Wu et al. (2010) studied the diversity of 36 cultivars and proved that the genetic relationship defined by molecular marker was basically consistent with the variety pedigree, but it could not be grouped only by pedigree or origins. The analysis of 56 sea-island cotton accessions in this study further confirmed this point, so it is important to combine genetic relationship by molecular markers and pedigree information to analyze genetic diversity.

Compared to the UPGMA dendrogram of SRAPs (Li et al., 2008), 37 (Xinhai5) was also grouped alone; however, 56 (Xinhai25) could be clustered with the latest cultivars from Xinjiang based on SSRs. Both SRAPs and SSRs showed that the latest cultivars from Xinjiang were clustered together, and the most basic ones from Xinjiang were clustered with the foreign ones. Some disagreements appeared between SSRs and SRAPs; for example, 6 (Giza47), 9 (Baghmaramian), 13 (Syriamian), 30 (Shenli1), 41 (Xinhai9), and 51 (Xinhai19) were clustered in different groups. Previous research also compared genetic diversity by analyzing these two kinds of markers. Although the 
results were different, the combined analysis of SSR and SRAP contained more abundant information (Zhang et al., 2007; Wang et al., 2008). As SSR and SRAP detect different variations of the genome, it is reasonable to obtain some different results. In order to obtain more comprehensive results, we combined the data of SSR and SRAP markers to get an integrated result as shown in Figure 4. In this Figure, the 56 accessions were divided into 4 groups: Xinhai5 and Xinhai25 were grouped, respectively, as group I and $\alpha$; later varieties in Xinjiang are clustered together as group $\beta$, and the rest of the accessions were group $\chi$. The results of UPGMA dendrogram based on SSRs+SRAPs (Figure 4) confirmed the necessity of combining SSR and SRAP markers.

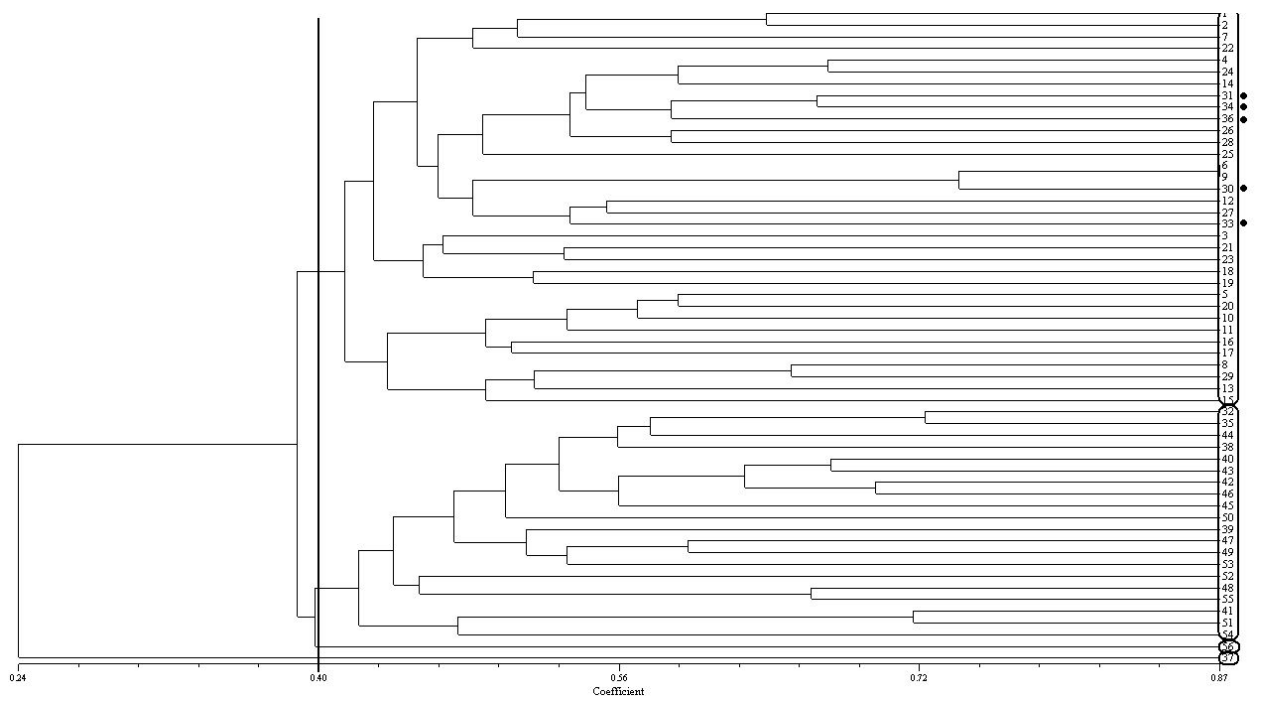

Figure 4. UPGMA dendrogram based on SSRs+SRAPs. Bold vertical line in the figure represents threshold lines. Oval boxes on the right represent different groups. The accessions are followed by a dot representing special individuals.

\section{ACKNOWLEDGMENTS}

Research supported by the National Science Foundation of China (\#30871559).

\section{REFERENCES}

Chen G, Du XM, Lu DB, Zhou ZL, et al. (2005). Genetic diversity of Sea Island cotton using SSR markers. J. Plant Genet. Resour. 6: 135-139.

Cruz VMV, Luhman R, Marek LF, Rife CL, et al. (2007). Characterization of flowering time and SSR marker analysis of spring and winter type Brassica napus L. germplasm. Euphytica 153: 43-57.

Diao M, Chu GX, Li SK and Cao LP (2002). Studies on the relationship of upland cotton varieties in the course of replacement of varieties in North Xinjiang over the past fifty years. Sci. Agr. Sin. 35: 1456-1460.

Dongre A, Parkhi V and Gahukar S (2004). Characterization of cotton (Gossypium hirsutum) germplasm by ISSR, RAPD markers agronomic values. J. Biot. 3: 388-393.

Frelichowski JE Jr, Palmer MB, Main D, Tomkins JP, et al. (2006). Cotton genome mapping with new microsatellites from Acala "Maxxa" BAC-ends. Mol. Genet. Genomics 275: 479-491.

Fryxell PA (1992). A revised taxonomic interpretation of Gossypium L. (Malvaceae). Rheedea 2: 108-165.

Guo WZ, Cai CP, Wang CB, Han ZG, et al. (2007). A microsatellite-based, gene-rich linkage map reveals genome structure, function, and evolution in Gossypium. Genetics 176: 527-541. 
He DH, Lin ZX, Zhang XL, Nie YC, et al. (2007). QTL mapping for economic traits based on a dense genetic map of cotton with PCR-based markers using the interspecific cross of Gossypium hirsutum x Gossypium barbadense. Euphytica 153: 181-197.

He LR, Zheng DM, Zhang XL, Zhang L et al. (2002). Analysis of pedigree and evolution of main agronomic traits of selfcultivated sea-island cotton in South Xinjiang. China Cotton 29: 8-9.

Iqbal MJ, Reddy OUK, El-Zik KM and Pepper AE (2001). A genetic bottleneck in the "evolution under domestication" of upland cotton Gossypium hirsutum L. examined using DNA fingerprinting. Theor. Appl. Genet. 103: 547-554.

Jiang W, Zhu HB and He JM (2008). Genetic diversity in germplasm resources of cotton from different area based on ISSR markers. Acta Agron. Sin. 20: 348-353.

Kantartzi SK, Ulloa M, Sacks E and Stewart JMD (2009). Assessing genetic diversity in Gossypium arboreum L. cultivars using genomic and EST-derived microsatellites. Genetica 136: 141-147.

Kong QP (2002). Overview of Sea-island cotton production and comparative advantage of China. China Cotton. 29: 19-20.

Lacape JM, Dessauw D, Rajab M, Noyer JL, et al. (2007). Microsatellite diversity in tetraploid Gossypium germplasm: assembling a highly informative genotyping set of cotton SSRs. Mol. Breed. 19: 45-58.

Li JR, Wang XG, Zhu YJ and Zhang W (2009). Cluster analysis on 14 Sea-island Cotton by SSRs. Xinjiang Agr. Sci. 46: 237-241.

Li W, Ni W, Lin ZX and Zhang XL (2008). Genetic diversity analysis of sea-Island cotton cultivars using SRAP markers. Acta Agron. Sin. 34: 893-898.

Luo R, Wu WL, Zhang Y and Li YH (2010). SSR marker and its application to crop genetics and breeding. Genomics Appl. Biol. 29: 137-143.

Mehetre SS, Aher AR, Gawande VL, Patil VR et al. (2003). Induced polyploidy in Gossypium: a tool to overcome interspecific incompatibility of cultivated tetraploid and diploid cottons. Curr. Sci. 84: 1510-1512.

Mei YJ, Ding S, Zheng DM, Cao XC et al. (2001). Analysis and evaluation of grey relational of south Sea-island cotton varieties (lines). China Cotton 28: 16-17.

Nguyen TB, Giband M, Brottier P, Risterucci AM, et al. (2004). Wide coverage of the tetraploid cotton genome using newly developed microsatellite markers. Theor. Appl. Genet. 109: 167-175.

Qureshi SN, Saha S, Kantety RV, Jenkins JN et al. (2004). EST-SSR: a new class of genetic markers in cotton. Cotton Sci. 8: 112-123.

Rana MK, Singh VP and Bhat KV (2005). Assessment of genetic diversity in upland cotton (Gossypium hirsutum L.) breeding lines by using amplified fragment length polymorphism (AFLP) markers and morphological characteristics. Genet. Resour. Crop Evol. 52: 989-997.

Rohlf FJ (2000). NTSYS-pc: Numerical Taxonomy and Multivariate Analysis System, Version 2.1. Exeter Software, New York.

Rong JK, Abbey C, Bowers JE, Brubaker CL et al. (2004). A 3347-locus genetic recombination map of sequence-tagged sites reveals features of genome organization, transmission and evolution of cotton (Gossypium). Genetics 389.

Song XL, Sun XZ, Zhang TZ and Wang HG (2004). Advances on genetic diversity of cotton (Gossypium). Acta Bot. Boreali-Occidentalia Sin. 24: 2393-2397.

Tan ZM, Li YC, Hu Q, Mei DS et al. (2009). Genetic diversity of parental lines of rapeseed hybrids based on SSR and SRAP markers. J. Agr. Biot. 15: 882-890.

Vafai-Tabar M, Chandrashekaran S, Rana MK and Bhat KV (2004). RAPD analysis of genetic diversity in Indian tetraploid and diploid cotton (Gossypium spp). J. Plant Biochem. Biot. 13: 81-84.

Wang HZ, Wu ZD, Wang XW and Fang ZY (2008). Analysis of the genetic diversity in different types of sugar beets by SRAP and SSR markers. Acta Agron. Sin. 34: 37-46.

Wang XL and Diao ZF (2002). Analysis of long staples cotton pedigree in Xinjiang. China Seeds 12: 21-22.

Westengen OT, Huaman Z and Heun M (2005). Genetic diversity and geographic pattern in early South American cotton domestication. Theor. Appl. Genet. 110: 392-402.

Wu DP, Fang XX, Ma MN, Chen JH et al. (2010). Genetic relationship and diversity of the germplasms in Gossypium barbadense L. from four different countries using SSR markers. Cotton Sci. 22: 104-109.

Yu JW, Yu SX, Lu C, Wang W et al. (2007). High-density linkage map of cultivated allotetraploid cotton based on SSR, TRAP, SRAP and AFLP markers. J. Integr. Plant Biol. 49: 716-724.

Zhang P, Zhang HY, Guo WZ, Zheng YZ et al. (2007). Genetic diversity analysis of Sesamum indicum L. germplasms using SRAP and EST-SSR markers. Acta Agron. Sin. 33: 1696-1702.

Zhang Y, Lin Z, Xia Q, Zhang M, et al. (2008). Characteristics and analysis of simple sequence repeats in the cotton genome based on a linkage map constructed from a $\mathrm{BC} 1$ population between Gossypium hirsutum and G. barbadense. Genome 51: 534-546.

\section{SUPPLEMENTARY MATERIAL}




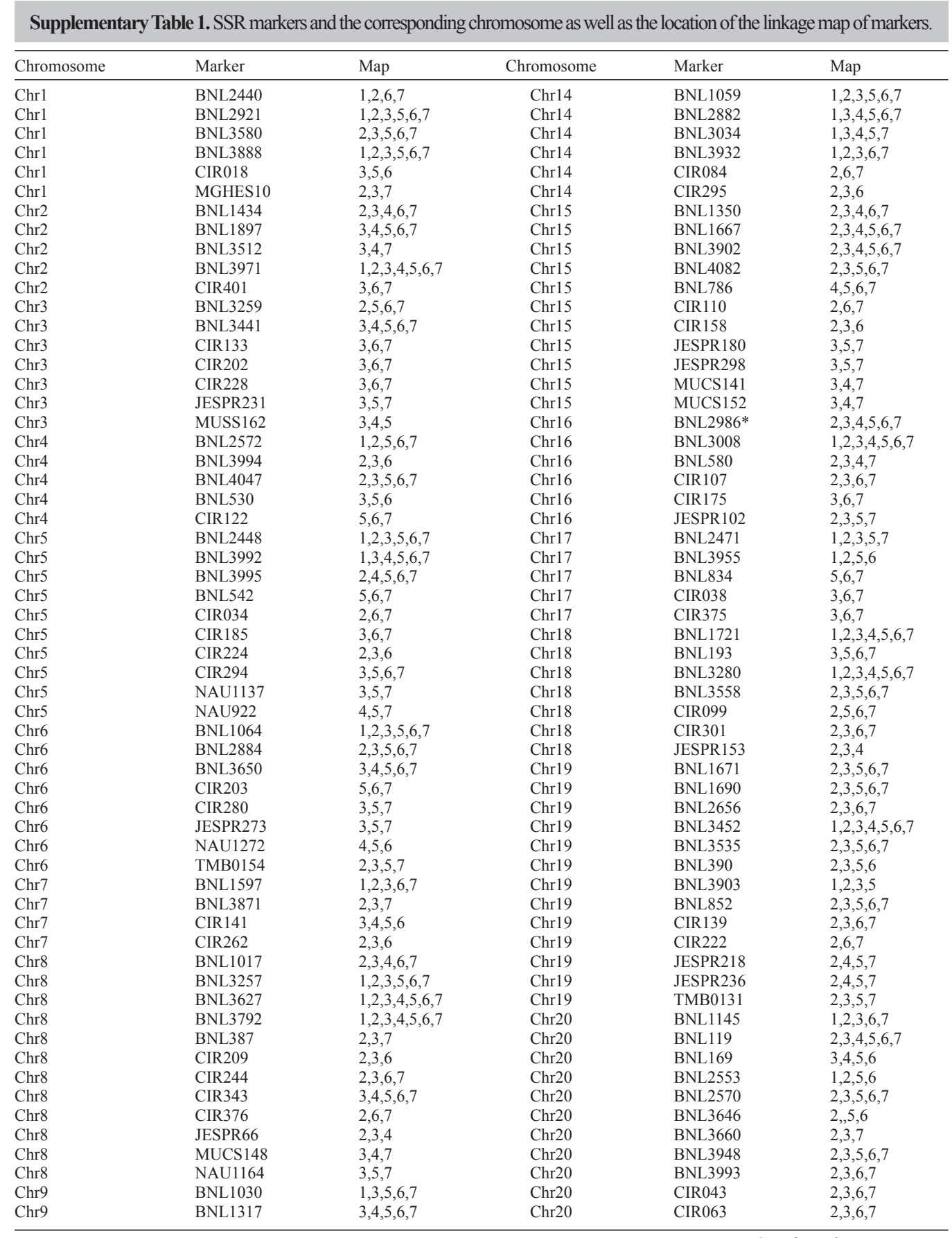

Continued on next page 


\begin{tabular}{|c|c|c|c|c|c|}
\hline Chromosome & Marker & Map & Chromosome & Marker & Map \\
\hline Chr9 & BNL1414 & $2,3,5,6,7$ & Chr20 & CIR121 & $2,3,6,7$ \\
\hline Chr9 & BNL1423 & $1,6,7$ & Chr20 & JESPR235 & $3,4,7$ \\
\hline Chr9 & BNL2847 & $2,5,6,7$ & Chr21 & BNL1551 & $5,6,7$ \\
\hline Chr9 & BNL3582 & $1,2,5,6,7$ & Chr21 & BNL3171 & $1,2,3,5,6,7$ \\
\hline Chr9 & BNL3626 & $1,5,7$ & Chr21 & BNL3279 & $3,5,6$ \\
\hline Chr9 & CIR019 & $2,5,7$ & Chr21 & BNL3449 & $2,3,5,7$ \\
\hline Chr9 & JESPR208 & $2,3,5,7$ & Chr21 & CIR068 & $2,3,5,6,7$ \\
\hline Chr9 & JESPR290 & $2,3,7$ & Chr21 & JESPR251 & $2,3,5$ \\
\hline Chr9 & MGHES70 & $2,3,7$ & $\mathrm{Chr} 23$ & BNL1672 & $2,3,5,6,7$ \\
\hline Chr10 & BNL1161 & $1,2,3,5,6$ & Chr23 & BNL2690 & $2,3,7$ \\
\hline Chr10 & BNL1665 & $2,3,4,5,7$ & Chr23 & BNL3511 & $2,3,5,6,7$ \\
\hline Chr10 & BNL2705 & $2,3,4,7$ & $\mathrm{Chr} 23$ & BNL686 & $2,3,5,6,7$ \\
\hline Chr10 & BNL3563 & $4,5,6,7$ & $\mathrm{Chr} 23$ & CIR060 & $2,3,5,6$ \\
\hline Chr10 & BNL3790 & $2,3,7$ & Chr23 & CIR286 & $3,5,6,7$ \\
\hline Chr10 & BNL3895 & $2,3,4,5,6,7$ & $\mathrm{Chr} 23$ & JESPR110 & $2,5,7$ \\
\hline Chr10 & CIR171 & $3,6,7$ & Chr23 & JESPR151 & $2,3,5$ \\
\hline Chr10 & TMB0307 & $2,3,5,7$ & Chr23 & JESPR274 & $3,5,7$ \\
\hline Chr10 & TMB0325 & $2,3,5$ & $\mathrm{Chr} 24$ & BNL1521 & $2,3,5,6,7$ \\
\hline Chr11 & BNL1034 & $1,2,3,4,6,7$ & $\mathrm{Chr} 24$ & BNL1646 & $5,6,7$ \\
\hline Chr11 & BNL1151 & $1,3,4,5,6,7$ & $\mathrm{Chr} 24$ & BNL252 & $3,6,7$ \\
\hline Chr11 & BNL1404 & $2,3,5,6$ & $\mathrm{Chr} 24$ & BNL2597 & $2,5,7$ \\
\hline Chr11 & BNL1681 & $2,5,6$ & $\mathrm{Chr} 24$ & BNL2961 & $1,2,6,7$ \\
\hline Chr11 & BNL2589 & $1,2,3,5,6,7$ & $\mathrm{Chr} 24$ & BNL3474 & $2,5,6,7$ \\
\hline Chr11 & BNL2632 & $1,2,3,5,6,7$ & Chr24 & BNL3860 & $1,2,5,6,7$ \\
\hline Chr11 & BNL2805 & $2,3,6,7$ & $\mathrm{Chr} 24$ & CIR026 & $3,5,7$ \\
\hline Chr11 & BNL3411 & $1,2,5,6,7$ & $\mathrm{Chr} 24$ & CIR413 & $2,5,6$ \\
\hline Chr11 & BNL3431 & $1,2,3,5,6,7$ & $\mathrm{Chr} 24$ & JESPR $157^{*}$ & $2,3,5,7$ \\
\hline Chr11 & BNL3442 & $1,2,3,5,6,7$ & $\mathrm{Chr} 24$ & TMB0555 & $2,3,5$ \\
\hline Chr11 & BNL3592 & $2,3,4,5,6,7$ & $\mathrm{Chr} 25$ & BNL1047 & $2,5,6,7$ \\
\hline Chr11 & BNL4094 & $2,3,5,6,7$ & Chr25 & BNL1153 & $1,3,5,6,7$ \\
\hline Chr11 & BNL625 & $3,4,5,6,7$ & $\mathrm{Chr} 25$ & BNL1417 & $4,6,7$ \\
\hline Chr11 & CIR003 & $4,6,7$ & $\mathrm{Chr} 25$ & BNL1440 & $1,2,3,5,6,7$ \\
\hline Chr11 & CIR254 & $3,6,7$ & $\mathrm{Chr} 25$ & BNL3103 & $2,3,4,5,6,7$ \\
\hline Chr11 & JESPR135 & $3,4,5,7$ & Chr25 & BNL3190 & $2,3,4$ \\
\hline Chr11 & JESPR296 & $3,5,7$ & Chr25 & BNL3264 & $2,3,5,7$ \\
\hline Chr12 & BNL1045 & $2,4,7$ & Chr25 & BNL3806 & $2,3,5,6,7$ \\
\hline Chr12 & BNL1673 & $3,5,6,7$ & Chr25 & CIR109 & $2,3,6,7$ \\
\hline Chr12 & BNL1707 & $2,5,6,7$ & $\mathrm{Chr} 25$ & CIR 150 & $2,3,5,6$ \\
\hline Chr12 & BNL2709 & $3,4,7$ & Chr25 & CIR298 & $3,6,7$ \\
\hline Chr12 & BNL2967* & $2,3,6$ & Chr 25 & CIR407 & $2,3,5,6,7$ \\
\hline Chr12 & BNL3261 & $2,5,6,7$ & Chr25 & JESPR 215 & $2,3,5,7$ \\
\hline Chr12 & BNL3599 & $3,5,6,7$ & Chr25 & JESPR 224 & $2,4,5$ \\
\hline Chr12 & BNL3835 & $3,6,7$ & Chr25 & JESPR227 & $2,3,5,7$ \\
\hline Chr12 & BNL4059 & $1,2,3,5,6,7$ & Chr25 & JESPR229 & $2,3,4,5$ \\
\hline Chr12 & CIR081 & $3,5,6$ & Chr25 & TMB0313 & $2,5,7$ \\
\hline Chr12 & CIR293 & $3,6,7$ & $\mathrm{Chr} 25$ & TMB0436 & $2,5,7$ \\
\hline Chr12 & CIR362 & $3,5,6,7$ & Chr26 & BNL1669 & $2,3,5,6,7$ \\
\hline Chr12 & NAU1237 & $4,5,7$ & $\mathrm{Chr} 26$ & BNL2495 & $1,2,3,5,6,7$ \\
\hline Chr12 & NAU1301 & $3,5,7$ & Chr26 & BNL2725 & $2,3,4,7$ \\
\hline Chr13 & BNL1421 & $4,5,6,7$ & Chr26 & BNL3368 & $1,2,3,7$ \\
\hline Chr13 & BNL1438 & $2,3,5,6,7$ & Chr26 & BNL341 & $2,3,4,5,6,7$ \\
\hline Chr13 & BNL1495 & $4,5,6,7$ & Chr26 & BNL3482 & $2,5,6,7$ \\
\hline Chr13 & BNL2449 & $3,5,6,7$ & Chr26 & BNL3510 & $2,3,4,5,6,7$ \\
\hline Chr13 & BNL2652 & $1,2,5,6$ & Chr26 & BNL3537 & $2,5,6,7$ \\
\hline Chr13 & BNL4029 & $3,5,7$ & Chr26 & BNL3816 & $2,4,5$ \\
\hline Chr13 & BNL409 & $3,5,6$ & Chr26 & CIR032 & $2,6,7$ \\
\hline Chr13 & CIR054 & $2,3,5,6$ & Chr26 & CIR085 & $2,5,6$ \\
\hline Chr13 & CIR096 & $2,4,6$ & Chr26 & MUSS303 & $3,4,5$ \\
\hline Chr13 & CIR342 & $5,6,7$ & $\mathrm{Chr} 26$ & NAU1231 & $4,5,7$ \\
\hline Chr13 & CIR406 & $2,3,6,7$ & & & \\
\hline
\end{tabular}
et al., 2004); 7 (Yu et al., 2007). 


\begin{tabular}{|c|c|c|c|c|c|}
\hline Chromosome & Number of markers & Polymorphic rate $(\%)$ & Average amplified loci & Range of PIC value & Mean PIC value \\
\hline Chr1 & 7 & 85.7 & 2.17 & $0.035-0.761$ & 0.415 \\
\hline $\mathrm{Chr} 2$ & 4 & 75 & 1.00 & $0.035-0.462$ & 0.180 \\
\hline $\mathrm{Chr} 3$ & 7 & 100 & 1.43 & $0.035-0.726$ & 0.295 \\
\hline Chr4 & 5 & 100 & 1.80 & $0.035-0.748$ & 0.438 \\
\hline Chr5 & 10 & 100 & 1.40 & $0.035-0.623$ & 0.264 \\
\hline Chr6 & 8 & 50 & 1.75 & $0.035-0.734$ & 0.405 \\
\hline Chr7 & 4 & 100 & 1.52 & $0.035-0.408$ & 0.128 \\
\hline Chr8 & 12 & 91.8 & 1.91 & $0.035-0.816$ & 0.451 \\
\hline Chr9 & 11 & 100 & 1.80 & $0.035-0.845$ & 0.264 \\
\hline Chr10 & 9 & 100 & 2.56 & $0.035-0.855$ & 0.589 \\
\hline Chr11 & 17 & 100 & 1.41 & $0.035-0.777$ & 0.257 \\
\hline Chr12 & 15 & 93.4 & 1.36 & $0.035-0.796$ & 0.266 \\
\hline Chr13 & 11 & 81.8 & 1.78 & $0.035-0.851$ & 0.274 \\
\hline Chr14 & 8 & 87.5 & 1.29 & $0.035-0.614$ & 0.374 \\
\hline Chr15 & 10 & 100 & 1.73 & $0.035-0.762$ & 0.364 \\
\hline Chr16 & 5 & 100 & 1.40 & $0.035-0.628$ & 0.346 \\
\hline Chr17 & 5 & 100 & 1.60 & $0.035-0.734$ & 0.284 \\
\hline Chr18 & 7 & 85.7 & 1.33 & $0.035-0.611$ & 0.318 \\
\hline Chr19 & 13 & 92.3 & 1.92 & $0.035-0.783$ & 0.404 \\
\hline Chr20 & 13 & 92.3 & 1.75 & $0.035-0.742$ & 0.429 \\
\hline Chr21 & 6 & 100 & 2.00 & $0.069-0.672$ & 0.465 \\
\hline Chr23 & 9 & 100 & 1.56 & $0.035-0.783$ & 0.361 \\
\hline Chr24 & 11 & 81.8 & 1.78 & $0.035-0.818$ & 0.407 \\
\hline Chr25 & 17 & 88.3 & 1.53 & $0.035-0.788$ & 0.314 \\
\hline Chr26 & 13 & 92.3 & 1.67 & $0.035-0.778$ & 0.346 \\
\hline
\end{tabular}

$\mathrm{PIC}=$ polymorphism information content. 